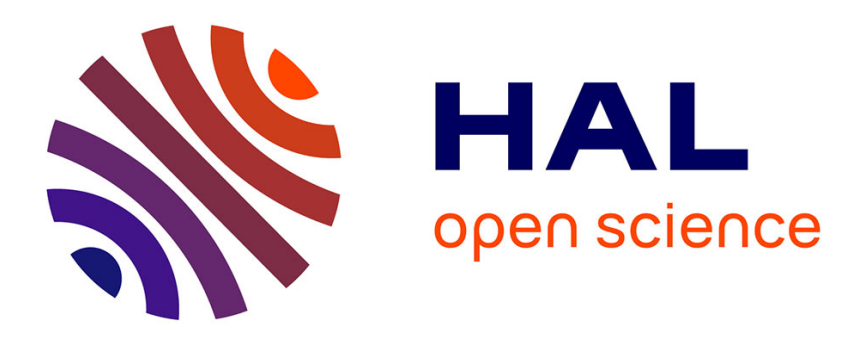

\title{
Fausse creance, mauvaise loi et conversion dans Perlesvaus
}

\author{
Christine Ferlampin-Acher
}

\section{To cite this version:}

Christine Ferlampin-Acher. Fausse creance, mauvaise loi et conversion dans Perlesvaus. Le Moyen Age. Revue d'histoire et de philologie, 2005, 111, p. 293-312. hal-01845685

\section{HAL Id: hal-01845685 \\ https://hal.science/hal-01845685}

Submitted on $20 \mathrm{Jul} 2018$

HAL is a multi-disciplinary open access archive for the deposit and dissemination of scientific research documents, whether they are published or not. The documents may come from teaching and research institutions in France or abroad, or from public or private research centers.
L'archive ouverte pluridisciplinaire HAL, est destinée au dépôt et à la diffusion de documents scientifiques de niveau recherche, publiés ou non, émanant des établissements d'enseignement et de recherche français ou étrangers, des laboratoires publics ou privés. 


\section{Ferlampin-Acher \\ Université de Rennes II}

Fausse creance, mauvaise loi et conversion dans Perlesvaus

dans Le Moyen Age, t. 111, 2005, p. 293-312 ;

Perlesvaus, roman en prose anonyme, se présente comme une suite du Conte du Graal de Chrétien de Troyes, Perlesvaus doublant Perceval dans sa quête du saint vessel. Ce récit a surpris les lecteurs modernes par la violence de certaines scènes d'anthropophagie ou de mutilations, contrastant avec la puissante christianisation de ce haut livre du Graal: R. S. Loomis allait jusqu'à émettre l'hypothèse que l'auteur devait souffrir d'une maladie mentale, paranoïa ou schizophrénie, et Helen Adolf expliquait cet étrange mélange de raffinement et de violence par l'existence de deux auteurs ${ }^{1}$. Mon projet est de montrer, à partir de la représentation des adversaires de la Chrétienté, Juifs, Sarrasins, païens, confondus dans la même altérité diabolique, comment l'unité de Perlesvaus se construit autour de l'idée de conversion ${ }^{2}$, sans qu'un arrière-plan idéologique

\footnotetext{
${ }^{1}$ R. S. Loomis, The Grail: from Celtic Myth to Christian Symbol, New York, 1927, p. 100 et H. Adolph, Visio Paci, State College, 1960, p. 125. Ces deux opinions sont rapprochées par Th. Kelly dans Le Haut Livre du Graal: Perlesvaus, Genève, Droz, 1974, p. 21-22. Je remercie Th. Kelly de m'avoir fait partager ses connaissances et ses réflexions sur ce texte étrange à l'occasion du colloque sur "Le monde païen dans l'historiographie et les textes littéraires du Moyen Age" (organisé par le Centre d'Etudes Polonaises et le Centre d'Etudes médiévales (Ph. Ménard), Paris, Sorbonne, 18 et 19 mai 2001), au cours duquel j'ai prononcé une communication qui me servit de point de départ à cet article. En ce qui concerne la cruauté de Perlesvaus, Ch. Méla ouvre les pages inspirées qu'il consacre à ce roman par des remarques sur le malaise qu'il suscite chez son lecteur (La reine et le Graal, Paris, Seuil, 1984, p. 176). Les citations de Perlesvaus sont faites à partir du texte donné dans le tome I de l'édition W. A. Nitze et T. Atkinson Jenkins, New York, Phaeton Press, 1972, 2 vol..

${ }^{2}$ L'importance du thème de la conversion dans ce roman est fort bien souligné par A. Saly qui voit en Perlesvaus un "héros convertisseur" ("Perceval-Perlesvaus. La figure de Perceval dans le Haut livre du Graal", Tra Li Li, XXIV, 2, 1986, p. 7-18, repris dans Image, Structure et Sens. Etudes arthuriennes, Senefiance, t. 34, 1994, p. 149-ss, l'expression figure p. 157). Dans "Le Perlesvaus et Gerbert de Montreuil", dans Miscellanea Mediaevalia, Mélanges
} 
ferme soit discernable (contrairement à ce que l'on a dans La Queste del Saint Graal avec laquelle Perlesvaus est fréquemment comparé). La notion de "conversion" serait plus de l'ordre de l'image obsessionnelle que de la réflexion spirituelle: en dépit de leur présence notable dans la deuxième partie du roman, les conversions restent limitées, ce que l'on pourrait rapprocher du fait que ce récit constitue une impasse pour le roman arthurien en prose, qui se développera moins à partir d'un Perlesvaus, resté, ce qui est étrange pour un texte aussi ancien, sans postérité, qu'à partir du Merlin en prose attribué à Robert de Boron.

\section{Les trois lois dans Perlesvaus}

Perlesvaus reprend l'opposition traditionnelle entre la Viez Loi et la Nouvele Loi ${ }^{3}$, mais lui donne, comme souvent au Moyen Age, une large extension, regroupant sous la dénomination Viez Loi des traits qui renvoient au judaïsme, conformément au sens premier de l'expression, mais aussi à la religion musulmane des sarrasins et au paganisme de l'Antiquité, cette dernière assimilation étant favorisée par l'ambiguïté médiévale du terme païen qui désigne aussi bien le musulman que le polythéiste des temps anciens, confondus dans une méconnaissance volontaire de l'Islam ${ }^{4}$. La scène d'ouverture de la branche II semble pourtant marquer une hiérarchie entre les Juifs et les Sarrasins: un jour de Saint-Jean, trois demoiselles arrivent à la cour d'Arthur, elles révèlent l'échec de Perceval au château du Graal, cause de la langeur du Roi Pécheur. Ces demoiselles sont accompagnées par un char, mené par trois cerfs blancs et contenant cent cinquante têtes de chevaliers, dont un tiers sont scellées d'or, un tiers, d'argent, un tiers, de plomb $^{5}$. L'aventure est glosée plus loin par un prêtre au Château de l'Enquête: li chief des

offerts à Philippe Ménard, Paris, Champion, 1998, t. II, p. 1163-ss, A. Saly montre que le motif de la conversion, anecdotique chez Gerbert, est dans Perlesvaus un élément fondamental, ce qui la conduit, à l'opposé de Nitze, à penser que Perlesvaus est postérieur à la Continuation de Gerbert.

${ }^{3}$ T. I, ligne 5419.

${ }^{4}$ Voir T. E. Kelly, op. cit., p. 101 ("the elastic quality of the term Old Law").

${ }^{5}$ P. 51. 
chevaliers seelé en or senefient la Novele Loi, et li chief seelé en argent les Giués, et li chief seelé en plon la fause loi des Sarazins ${ }^{6}$. L'argent a plus de valeur que le plomb: la primauté de l'Ancienne Loi des Juifs sur la fause loi des Sarazins vient de ce qu'elle annonce le Nouveau Testament. Cependant cette distinction n'est que théorique et la suite du roman entretient une confusion permanente entre la loi des Juifs et celle des Sarrasins ${ }^{7}$, confusion parfaitement illustrée par le sort réservé à ces têtes: celles des bons chrétiens finiront dans l'Isle Plenteürose, qui représente le Paradis, celles des Juifs et des Sarrasins, sans distinction, seront emportées par le Noir Ermite, Lucifer, dans l'infernale Isle Souffroiteuse ${ }^{8}$. D'une tripartition religieuse, on aboutit à une bipartition qui ne se révélera pas dépourvue de tout manichéisme ${ }^{9}$. Je chercherai donc dans un premier temps à cerner dans quelle mesure les diverses lois peuvent être identifiées.

Le roi Gurgaran ${ }^{10}$ est le premier "païen" à intervenir dans le roman. Le texte oppose explicitement sa loi et la loi des crestiens ${ }^{11}$ et les gens de son pays proient selonc leur creance ${ }^{12}$, sans plus de précision. Deux pratiques, en dépit de cette indétermination, retiennent l'attention du lecteur. Gurgaran fait cuire dans un vaissel d'airaim le cadavre de son fils tué par un géant et le partage entre ses hommes, puis il fait accrocher la tête du géant au-dessus de la porte. R. S. Loomis a rapproché cette anthropophagie d'une pratique païenne, rapportée aussi bien par Marco Polo que par Odoric de Pordenone ${ }^{13}$, au sujet d'un royaume qui reste difficile à identifier mais qui

\footnotetext{
${ }^{6}$ P. 109.

${ }^{7}$ Cette confusion est fréquente au Moyen Age: voir Ph. Sénac, L'image de l'autre. Histoire de l'occident médiéval face à l'Islam, Paris, Flammarion, 1978, p. 37-ss.

8 P. 399.

${ }^{9}$ Retenons la citation de J. C. Payen, pour qui "il n'y a d'autre idéologie, dans le Perlesvaus, qu'un manichéisme sommaire qui oppose deux mondes: le monde des païens, confondu avec celui du crime et de la cruauté, et le monde chrétien, où il est fait un devoir aux héros de répondre au sang par le sang et à l'horreur par l'horreur" (Le Motif du repentir dans la littérature française médiévale, Genève, Droz, 1967, p. 428).

10 P. 101-SS.

${ }^{11}$ Ligne 1995.

${ }^{12}$ Ligne 2025.

13 "Some additional sources of Perlesvaus", dans Romania, t. 81, 1960, p. 496.
} 
évoque un paganisme contemporain et exotique. Le lecteur médiéval pouvait aussi y "reconnaître" une pratique sarrasine, puisque dans Fierabras l'émir Balan imagine de rôtir sa propre fille. Cependant, le souci de l'auteur de préfigurer, avant la conversion de ce païen, le rite eucharistique $^{14}$, invite à voir dans ce récit plus une construction allégorisante qu'un témoignage anthropologique (même indirect). La coutume d'accrocher des têtes est tout aussi ambiguë. On la trouve au Moyen Age à la fois dans les récits d'inspiration celtique qui ont pu servir de base aux aventures arthuriennes ${ }^{15}$ et dans les récits de croisade ${ }^{16}$ : le motif tire les païens aussi bien du côté de Bran que d'Apolin et Mahomet. Aucun indice ne permet donc de renvoyer à une religion précise: le lecteur peut retrouver dans Gurgaran les sarrasins aussi bien que les païens d'Orient ou des anciens temps celtiques.

Si Gurgaran n'est qu'une figure passagère dans le roman, l'ombre du roi de Chastel Mortel plane de la branche I à la branche IX: l'on pourrait espérer trouver à son sujet des indices plus nombreux. On apprend qu'il est plein de felonie ${ }^{17}$, crueulx $^{18}$, qu'il est l'oncle de Perlesvaus par sa

\footnotetext{
${ }^{14}$ Sur la présence de l'eucharistie dans Perlesvaus, voir Ch. Méla, op. cit., p. 197 et W. Roach, "Eucharistic Tradition in the Perlesvaus", dans Zeitschrift für Romanische Philologie, LIX (1), 1939, p. 51-ss. C'est dans la même perspective que l'épisode consacré à Gurgaran s'ouvre sur une représentation allégorique de la Trinité (trois demoiselles semblent à Gauvain n'en être qu'une).

${ }^{15}$ On trouve par exemple des têtes coupées dans Peredur: voir M. Williams, Essai sur la composition du roman gallois de Peredur, Paris, 1909, p. 46-ss. Voir A. Reinach, "Le rite des têtes coupées chez les Celtes", dans Revue de l'histoire des religions, t. 67, 1913, p. 41-48 et "Les têtes coupées et les trophées en Gaule", dans Revue celtique, t.
} 34, 1913, p. 38-60.

${ }^{16}$ Les chroniqueurs latins de la première croisade mentionnent souvent la coutume sarrasine d'exhiber les têtes des vaincus comme signe de victoire, que ces têtes soient empalées ou suspendues. Le motif est fréquent dans les chansons de geste (Les Saisnes, Floovant, La destruction de Rome...). Les traités relatifs à la guerre sainte islamique interdisent cependant cette pratique sauf par représailles ou pour abattre le moral des ennemis. Il est possible que la vogue épique des têtes coupées ait été renforcée par l'hagiographie. Sur les pratiques musulmanes, entre fantasme et réalité, voir P. Bancourt, Les musulmans dans les chansons de geste du cycle du roi, Aix-en-Provence, 1982, 2 vol. (t. I, p. 178 pour les têtes coupées). Dans La Vie de saint Louis de Joinville, le roi, pendant son séjour à Acre, demande aux messagers des émirs égyptiens de rendre toutes les testes des crestiens qui pendoient entour les murs du Kaire des le tens que le conte de Bar et le conte de Monfort furent pris (éd. J. Monfrin, Paris, Garnier, 1995, §469).

${ }^{17}$ L. 1082, 1. 3929. 
mère et qu'il a renoié Dieu ${ }^{19}$. Il s'est emparé des terres du roi Pécheur, et après la reconquête de celles-ci par Perlesvaus, il se suicide du haut des créneaux de sa ville ${ }^{20}$. C'est donc un renégat, abandonné de Dieu au point d'attenter à sa vie. Il est néanmoins difficile de le situer: sa maîtrise de la mer (il se déplace en galie $^{2 l}$ ) renvoie aussi bien au monde sarrasin qu'aux incursions des barbares du nord de sinistre mémoire. De même, le Chevalier au Dragon vit dans l'Ile des Eléphants $^{22}$ : l'exotisme de son habitat ainsi que le caractère diabolique de son enseigne en font un mécréant, mais aucun indice précis n'est donné. La Reine au Cercle d'Or n'est pas non plus chrétienne: elle promet de croire en la loi des crestiens, en la loi qui novelement est establie (...): "Vostre loi vaut meuz que la nostre, et que Diex naqui de fame" ${ }^{23}$. L'adverbe novelement, la hiérarchie établie entre les deux religions et l'insistance sur la Nativité suggèrent qu'elle est juive, même si le texte entretient un relative indétermination. De même, le château de Grant Defois, qui tourne sur lui-même, est habité par des gens qui pratiquent la Viez $\mathrm{Loi}^{24}$, sans qu'aucun détail supplémentaire ne soit donné. On apprend qu'il a été fondé par Virgile, mais la fréquence de celui-ci dans les romans comme magicien, ne suffit pas à orienter vers le paganisme antique, d'autant qu'on lisait sa quatrième Bucolique comme une annonce du Christ sauveur.

Le Seigneur des Marés porte de même un nom des plus ambigus: il est le seigneur des Mares, Marés, ou des Mores, des Maures $^{25}$, l'imagination s'accommodant facilement de l'association entre la fange et les ennemis de Dieu. Adversaire de la mère de Perlesvaus, la Veuve Dame, il semble apparaître à deux reprises: d'abord (l'édition de Nitze transcrit alors Marés), il est

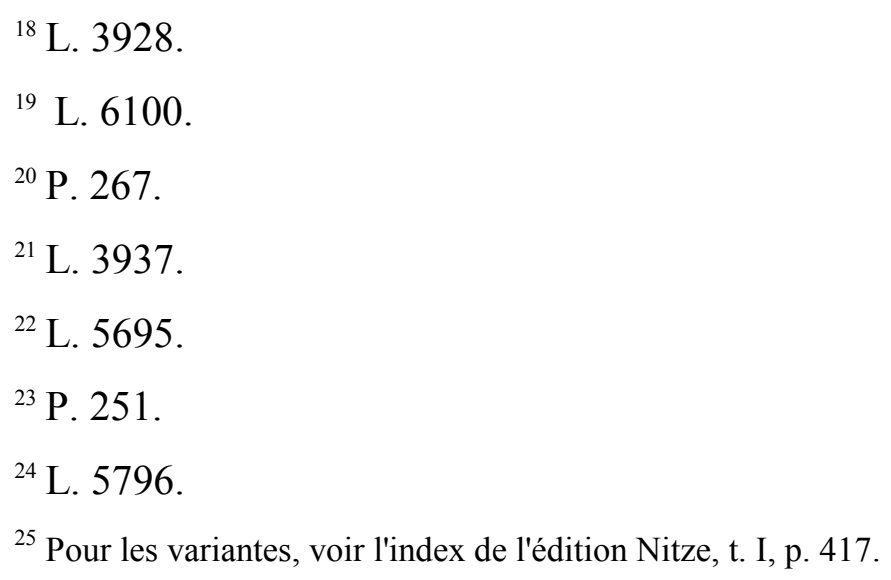


vaincu par Gauvain et promet de ne plus nuire à la Veuve Dame ${ }^{26}$. Il est présenté dans cet épisode tout au plus comme un félon (Gauvain lui reproche torz e pechiez ${ }^{27}$, ce que renforce son alliance avec Kahos li Rox, à la rousseur diabolique. Plus loin, on retrouve un seigneur des Mores, que l'index identifie avec le premier: lui aussi est hostile à la Dame ; lui aussi est d'abord présenté par la soeur de Perlesvaus ${ }^{28}$. Le goût de l'auteur pour les duplications est confirmé par cet exemple, mais, en dépit de l'identification suggérée par l'index entre les deux personnages, la divergence des noms et l'absence de corrélation explicite entre les deux épisodes, n'excluent pas qu'ils soient différents, même si une dernière occurrence, dans la bouche de la mère ${ }^{29}$ nomme "des Marez" l'adversaire tué par Perlesvaus ${ }^{30}$. Quoi qu'il en soit, rien n'est dit quant à sa (leur) foi. Perlesvaus condamne le seigneur des Mores à une mort étrange (il le noie dans une cuve de sang), après avoir invoqué la Viez Loi et la Nouvele qui commandent, dit-il, que l'en feïst justice des omicides et des traïtors ${ }^{31}$, suggérant peut-être que le seigneur de Mores est Juif et qu'il le condamne autant au nom de sa propre loi qu'en celui de la loi des Chrétiens.

Alistor (ou Aristor) d'Amorave porte un nom qui souligne son étrangeté: Ali- fonctionne souvent comme préfixe marquant l'altérité ${ }^{32}$, Amorave suggère un exotisme peu défini géographiquement. Frère du seigneur des Marés, c'est une sorte de Barbe-Bleue qui tue

${ }^{26}$ P. 72.

${ }^{27}$ L. 1137.

${ }^{28}$ P. 227.

${ }^{29}$ P. 306.

${ }^{30}$ Si une étude des variantes des noms de ces deux (?) figures dans les divers manuscrits permettrait peut-être d'élucider ce point, le texte présenté par Nitze, convergence des lectures successives par les copistes et l'éditeur, provoque chez le lecteur l'impression d'abord de deux personnages différents, avant qu'in extremis l'intervention de la Veuve Dame ne vienne brouiller les pistes, proposant une identité que rien par ailleurs ne suggérait jusque-là dans le texte.

${ }^{31}$ P. 234.

${ }^{32}$ Voir les Alimagot et autres Alipatin, qu'Al(i)- renvoie pour le lecteur médiéval au latin ou à l'arabe. 
régulièrement sa nouvelle épouse au bout d'un an en lui coupant la tête ${ }^{33}$. Après avoir tué le Roi Pécheur, il veut épouser la soeur de Perlesvaus de force. Mais à part sa cruauté et sa felonie ${ }^{34}$, on n'en apprend rien, et Perlesvaus, expéditif, lui coupe la tête ${ }^{35}$, sans qu'il ait le temps d'invoquer un Dieu quelconque ${ }^{36}$.

Outre ces renvois aussi divers qu'indéterminés, la mention du diable dispense en général l'auteur de toute précision: le seigneur du Chastel de Grant Defois porte l'esperit du deable ${ }^{37}$; pour le Noir Ermite, ultime adversaire de Perlesvaus, la répétition des adjectifs noir, led, hideus et la comparaison com se ce fust enfer, suffisent à évoquer l'Enfer et le Diable ${ }^{38}$. Par ailleurs la technique de présentation de ces personnages rend vaine toute identification. En effet les païens sont avant tout des sujets de discours, leur fame l'emporte sur l'évocation directe de ce qu'ils sont. Toujours précédés par leur réputation d'Infidèles, énoncée par des informateurs bons chrétiens ${ }^{39}$, ils sont définis essentiellement par cette hostilité au christianisme, qui, une fois posée, n'a besoin d'être confirmée dans les scènes où ces païens paraissent enfin que par des détails épars et peu nombreux. Viennent ensuite des gloses, par des sages, qui, après l'éradication de ces mécréants, corroborent l'interprétation à donner à l'épisode: les païens ne valent alors que comme faire-valoir d'un catéchisme, peu précis, mais efficace. Le culte des autres n'intéresse pas en tant que tel. Dans les cités où règnent les coutumes les plus barbares, Château des Barbes ou Gaste Cité du Jeu du

33 P. 306. Ce motif témoigne d'une intéressante combinaison: on y retrouve le motif des royautés temporaires, au terme desquelles le héros est supposé se faire couper la tête, ainsi qu'une déformation de la polygamie musulmane (dans Floire et Blancheflor de même l'émir prend une nouvelle femme chaque année: voir P. Sénac, op. cit., p. 86).

${ }^{34}$ L. 8749.

${ }^{35}$ Cette mort infamante revient logiquement à ce païen, fervent adepte de la décollation féminine.

${ }^{36}$ On en apprend aussi peu sur le Roux Chevalier et sur le Chevalier de la Galie. Celui-ci pend les Chrétiens (p. $377-$ ss), il est cruel (1. 9284), il a tué le Roi Ermite, et il veut contraindre Célestre, nouvellement baptisée, à renier Dieu (1. 9302). Diabolique, comparé avec la tempeste (1. 9321), il a la tête coupée par Meliot en quelques lignes.

37 L. 5763.

${ }^{38}$ P. 402-SS.

39 Ainsi les méfaits du seigneur des Marés sont d'abord évoqués par la soeur de Perlesvaus (p. 43), puis par sa mère (p. 67). 
Décapité, aucune référence précise n'est faite à la religion: il est évident pour l'auteur et il doit l'être pour le lecteur, que ces espaces ne sont pas chrétiens et il est sans intérêt de peindre leur loi puisque seule la relation d'hostilité vaut. Que ces lieux ne sont pas chrétiens est néanmoins parfois suggéré par quelques indices concernant directement la religion: lorsque Lancelot revient à la Gaste Cité pour se soumettre au jeu du décapité, il communie par l'herbe, comme de nombreux héros épiques ${ }^{40}$ : c'est bien la confirmation que cette cité est sans prêtre ni croix ${ }^{41}$.

Les aventures, en particulier celles qui sont réservées à Perlesvaus, sont donc en général présentées d'abord par des messagers ou des informateurs, qui résument la situation en mentionnant le danger que court la Nouvelle Loi aux prises avec l'Ancienne Loi. Chaque confrontation avec des mécréants est donc préjugée par la présentation qui la précède: ainsi chez la Veuve Dame, la Demoiselle du Char explique à Perlesvaus que le roi de Chastel Mortel veut convertir les habitants de la terre du roi Pécheur à l'Ancienne Loi; ainsi le messager de Madaglan d'Orande explique la situation de Jandree. Les gloses, postérieures aux événements, commentent ceux-ci en insistant sur le conflit entre les lois et ne sont que des confirmations. Dans le livre VI, au Château de l'Enquête, Gauvain apprend par le tesmoignage de Josep le bon clerc et le bon hermite la signification des têtes scellées d'or, d'argent et de plomb; le Noir Ermite est Lucifer, la Demoiselle Chauve, Fortune, qui, chauve avant la Crucifixion et chevelue après, incarne le passage de l'Ancienne à la Nouvelle Loi. Gauvain interroge ensuite le prêtre sur la dame tuée par son mari: elle symbolise la Viez Loi qui fu abatue par un coup de glaive sanz resociter, et por la Viez Loi se sofri Diex a ferir en coste du glaive, et par ce coup fu la Viez Loi abatue et par son crucefiement. La dame senifie la Viez $\mathrm{Loi}^{42}$. Cette lecture semble satisfaire Gauvain qui, sans commentaires, enchaîne sur le chevalier couard, Meliot et l'ymaige qui s'est cachée dans la fontaine sur son passage.

Le problème le plus grave que révèlent ces commentaires, qu'ils soient antérieurs ou postérieurs aux événements, semble être celui de l'engendrement de la Nouvelle Loi par

\footnotetext{
${ }^{40}$ Sur la communion par l'herbe, voir J. C. Payen, op. cit., p. 227.

${ }^{41}$ Ibid., p. 432.

${ }^{42}$ L. 2208-ss.
} 
l'Ancienne. Si l'altérité radicale des divers païens ne pose finalement guère de problème dans Perlesvaus tant leur caractère diabolique est évident, le rapport génétique et historique entre l'ancienne et la nouvelle loi reste épineux et les commentaires reviennent sans cesse sur la figure du Christ où s'incarne le passage entre les deux temps. La rencontre entre Meliot et Gauvain est selon Jean Frappier une "leçon de catéchisme en image et en mouvement" ${ }^{43}$ centrée sur la figure du Sauveur, représenté par Meliot, monté sur un lion. La glose insiste sur le fait qu'il est né dans ${\text { l'Ancienne } \text { Loi }^{44} \text {, figurée par sa mère }}^{45}$. Le commentaire concernant sa mère et la loi des Juifs est donné avant celui qui porte sur le Fils et la Nouvelle Loi ${ }^{46}$ : la structure et l'allégorie se rencontrent pour illustrer ce passage douloureux et incertain d'une loi à l'autre et l'intérêt porté à Meliot est significatif du caractère crucial de la réflexion sur le lien génétique entre l'Ancienne et la Nouvelle Loi. De la même façon, la Beste Glatissant est commentée par le Roi Ermite en fonction du lien historique entre les deux lois: la beste représente Nostre Seignor tandis que les douze chiens qui glapissent dans son ventre, progéniture immonde, sont les Jü̈s de la Viez Loi, tous fils ingrats ${ }^{47}$. Plus que l'étrangeté païenne, c'est donc la filiation entre les Juifs et les Chrétiens qui inquiète, ce dont témoigne l'ambiguïté d'un Madaglan d'Orande, mécréant qui rejette la Novele Loi tout en appartenant à la famille de la reine Guenièvre ${ }^{48}$, ou d'un roi du Chastel Mortel, oncle de Perlesvaus et néanmoins sectateur de l'Ancienne Loi ${ }^{49}$. Cette parenté entre l'Ancienne et la Nouvelle Loi est associée au lien lignagier qui unit les personnages, lien fondamental dans Perlesvaus où, pour reprendre l'expression de T. E. Kelly, le Graal ressemble à un secret de famille et où les créatures du Mal (comme celles du Bien) semblent toutes parentes ${ }^{50}$.

\footnotetext{
43 "Le Conte du Graal est-il une allégorie judéo-chrétienne?", dans Romance Philology, t. XX, 1966, p. 29.

44 P. 111.

${ }^{45}$ L. 2211.

${ }^{46}$ L. 2231.

${ }^{47}$ P. 257.

${ }^{48}$ L. 7848 et 1.7845 .

${ }^{49}$ L. 5420-ss.

${ }^{50}$ Op. cit., p. 52.
} 
Les gloses, qui pointent clairement les zones d'ombre, confirment que l'altérité des païens ne pose pas vraiment de problème, contrairement à la douloureuse parenté avec l'Ancienne Loi, favorisant l'apostasie, fréquente dans le roman. Cette proximité fait qu'il est nécessaire de renforcer l'altérité de l'Ancienne Loi pour que la condamnation soit sans ambiguïté: d'où la volontaire confusion entre Sarrasins, Païens et Juifs, d'où aussi des scènes d'une grande barbarie, comme celle où Perlesvaus noie le seigneur des Mores dans une cuve de sang. C'est parce qu'il est consanguin que l'univers de Perlesvaus doit être sanglant.

A la fin du roman, Perlesvaus et Lancelot sont confrontés à des figures qui sont clairement païennes, Jandrée et la dame du Chastel Enragié. Jandrée (par ailleurs soeur de Madaglan) somme Arthur de guerpir la Novele Loi, et de croire es Diex ou ele croit ${ }^{51}$. Le pluriel du substantif dieus $^{52}$ dénonce le polythéisme de la reine. Par ailleurs Jandrée a un tel desdaing pour ceux qui pratiquent la Nouvelle Loi qu'elle fait ses ieuz couvrir afin de ne pas les voir ${ }^{53}$ : le lecteur médiéval y reconnaîtra peut-être les femmes voilées de l'Islam ${ }^{54}$ tout en glosant, à la suite de Perlesvaus, l'aveuglement physique comme symbole des ténèbres spirituels où vit la mécréante ${ }^{55}$. Jandrée adore des dieux qui sont les doubles sombres du Dieu chrétien: ils ont accompli le miracle de la rendre aveugle, afin qu'elle n'ait plus besoin d'un voile pour échapper à la vue des Chrétiens, inversant le miracle chrétien traditionnel qui rend la vue aux aveugles. Avant de rencontrer Jandrée, conformément au principe de duplication des figures qu'affectionne particulièrement l'auteur de Perlesvaus, le héros est confronté à sa soeur, anonyme, son double en paganisme. Elle est idolâtre et vit la ou Dex n'estoit ne creüz ne amez, ainz aoroient fauses ymages e creoient dex qui onques ne furent. Deu ne pooient il mie estre, ainz estoient deable qui $s^{\prime}$ aparoient a els ${ }^{56}$. Son château est anciens tout comme celui de sa soeur ${ }^{57}$ : ce détail présente

\footnotetext{
${ }^{51}$ P. 330.

${ }^{52}$ Le pluriel est attesté à plusieurs reprises $(1.7934,1.7943,1.7950)$.

${ }^{53}$ L. 7942.

${ }^{54}$ Sur le voile qui intrigue les Occidentaux, voir Ph. Sénac, op. cit., p. 93.

${ }^{55}$ L. 7958.

${ }^{56}$ P. 369.
} 
l'idolâtrie de ces païens polythéistes comme un culte remontant à un passé ténébreux, par ailleurs limité à une $11 e^{58}$, même si les références possibles au monde sarrasin sont relativement claires et nombreuses (Perlesvaus rencontre un prisonnier qui porte un carcan au cou, qui renvoie au sort des esclaves qui émut tant la Chrétienté, les seigneurs du lieu jouent aux échecs, tout comme le géant qui a enlevé le fils de Gurgaran). La mauvaise loi est métaphorisée par l'aveuglement des femmes et la folie des hommes, elle est liée à l'animalisation et à la diabolisation: les seigneurs du Chastel Enragié deviennent forsené e enragié dès qu'ils voient des Chrétiens, ils braient come deable ${ }^{59}$. A la description précise des oppositions religieuses, l'auteur préfère la métaphore.

La confusion que nous constations entre sarrasins, païens, Juifs, semble finalement d'une part devoir être nuancée en fonction du moment où interviennent ces diverses figures dans le récit, et d'autre part correspondre à deux senefiances. Les figures clairement païennes interviennent soit tout au début du roman (avec la figure de Gurgaran), soit beaucoup plus loin, à la fin du récit (avec Jandrée et sa soeur par exemple). Entre-temps, c'est surtout à Gauvain et Lancelot que sont réservées les aventures centrées plus nettement sur l'opposition entre Chrétiens et Juifs. De là il ressort que l'enjeu suprême, revenant à la fin au héros Perlesvaus, est un enjeu de croisade, et qu'il est en quelque sorte subordonné à une lutte contre l'Ancienne Loi. Dans un premier temps, il faut lutter contre l'hérésie, la déviance, l'écart, contre ce qui est à la fois proche et différent: ce nos trete en senefiance li bons hermite por la Novele Loi, en laquele li plusor ne sont pas bien connoissant, si en volt fere remenbrance par essanples ${ }^{60}$. Le texte est alors riche en gloses qui problématisent le passage de l'Ancienne à la Nouvelle Loi, sa proche parente. Une fois clarifiée l'opposition entre la bonne et la mauvaise creance, Perlesvaus s'anime d'un esprit de croisade, tuant et convertissant, et les gloses, inutiles dans la mesure où l'altérité de la mauvaise loi est évidente, se font plus légères. La mission du chevalier est alors définie par Perlesvaus dans la dernière branche: il n'est nule si bele chevalerie come cele est que l'on fait por la loi Deu

\footnotetext{
${ }^{57}$ L. 9042 et 1.9165.

${ }^{58}$ L. 9047.

${ }^{59}$ L. 9103 et 1.9104.

${ }^{60}$ L. 2184-5.
} 
essaucier, e por lui se doit l'on miex pener que por tos les autres; autresi com il mist son cors en paine e en tavaill e en exill por nos, si doit chascuns le sien metre por lui" ${ }^{\prime 61}$. La succession des aventures correspond à deux enjeux différents: d'abord il s'agit de "renouveler" la méfiance envers l'Ancienne Loi, trop proche historiquement, avec des essamples qui ravivent la foi des personnages et des lecteurs et qui éveillent leur vigilance et évitent qu'ils ne succombent à une familiarité douteuse; le texte cherche alors à édifier, en insistant sur l'impossible conversion des mécréants; ensuite, il nourrit l'esprit de croisade dans des épisodes où certains infidèles sont convertis, sans que pour autant il soit nécessaire d'édifier le lecteur tant la cause est évidente. Dans le premier cas, la conversion aurait dû avoir lieu depuis la Crucifixion et n'est plus de saison, dans le second elle est encore possible. C'est donc autour de la conversion que s'opère une distinction forte (plus qu'au niveau des croyances): pour les Juifs la conversion aurait déjà dû s'accomplir, et plus que d'une conversion, il se serait agi d'une translatio, d'un passage, conformément au modèle si présent dans les imaginaires médiévaux, pour lesquels l'histoire est une translatio imperii, doublée d'une translatio studii et d'une translatio fidei ; pour les autres un retournement complet des croyances, une "conversion" est encore possible. C'est avec ceux dont la loi est la plus étrangère au christianisme que Perlesvaus se montrerait le moins "pessimiste" : la conversion serait moins incertaine que la translatio.

\section{Conversion et translatio dans Perlesvaus}

Se convertissent dans la branche VI Gurgaran (p. 105), dans la branche IX la reine au Cercle d'Or et son peuple (p. 253) et treize païens de la Tour de Cuivre (p. 255), dans la branche X les peuples conquis par Madaglan et regagnés par Perlesvaus (p. 351), dans la branche XI le Chastel Enragié et en particulier sa dame qui devient chrétienne sous le nom de Celestre (p. 373), ainsi que Jandree et les siens (p. 377) et le Chastel du Noir Ermite (p. 403). Mis à part l'épisode de Gurgaran, c'est à Perlesvaus que reviennent ces conversions.

${ }^{61}$ P. 370. 
On distinguera les conversions collectives et les conversions individuelles. Dans les deux cas, l'alternative épique (la conversion ou la mort) est mentionnée explicitement et la conversion du seigneur entraîne celle de l'ensemble de la population. Ces conversions ne retiennent guère l'auteur. La terre du Roi Pécheur, redevenue païenne sous l'influence du roi de Chastel Mortel, le mauvaiz roi qui la bone creance en avoit ostee (1. 6286), se convertit à nouveau une fois reconquise par Perlesvaus: il toli les vies a toz ceaus qui ne la voudrent ${ }^{62}$ croire. Li païs fu maintenus par lui e gardez, e la loi Nostre Seignor essauchie par sa force e par sa valor (p. 269). Le désintérêt est encore plus évident dans l'épisode du Château du Noir Ermite. Celui-ci, diabolique, ne s'est pas converti et sa chute dans un abîme sombre et puant a retenu l'auteur. En revanche, ce n'est qu'incidemment, une fois Perlesvaus sur le chemin du départ, que la conversion des gens du château est mentionnée, globalement (il n'est pas question de gens, mais du chastel par métonymie), avec une comparaison qui nivelle le caractère exceptionnel de l'aventure: Perlesvaus se parti du chastel joianz, car il l'ot atret a la creance Nostre Seigneur, e fist on son servise la dedenz autressi saintement com es autres lex (1. 10001-2).

Les conversions individuelles (qui en général entrainent des conversions collectives) sont plus intéressantes car plus développées. Gurgaran dans la branche VI (p. 105) est prédisposé à la conversion. Un géant a enlevé son fils et il ne trouve aucun chevalier assez hardi pour aller le sauver. Le roi en blasme plus assez sa loi que la loi des crestiens (1. 1995), et, espérant en la valeur de ces derniers, il les accueille fort bien. Par ailleurs Gurgaran est en possession de l'épée de quoi Saint Jehans fu decolez (1. 2008): comme le note J. C. Payen, les vertus des reliques favorisent la conversion. Enfin le peuple de Gurgaran paraît civilisé: tous prient, à leur façon certes, pour leur champion (1.2025) et manifestent une douleur profonde en découvrant la mort du fils du roi. La rupture entre ce deuil très chevaleresque et la scène de cannibalisme qui suit, où le père fait cuire le corps de son fils et le fait manger à ses gens n'est que sémantique, la syntaxe ne la laissant pas deviner: Il regraite son fil molt durement, et tuit cil del chastel. Après fet alumer .I. grant feu. Le lien simplement temporel gomme l'étrangeté de la scène et invite le lecteur à tempérer son horreur en transposant la lettre (Gurgaran préfigure l'Eucharistie, le rite païen

\footnotetext{
${ }^{62} \mathrm{La}$ renvoie à la Nouvelle Loi.
} 
annonce le culte chrétien, sur le mode de la translatio fidei). Le roi donne ensuite à Gauvain la relique sacrée qu'est l'épée, et demande le baptême, qu'il reçoit sous le nom d'Archier (p. 105). Gauvain coupe alors la tête de tous ceux qui ne veulent pas suivre leur seigneur. Vertus des reliques, importance des dispositions intérieures, possibilité d'une translatio religieuse, se retrouvent dans cette étrange scène de conversion. Il s'agit là d'une exception: d'une part c'est la seule conversion dont le mérite ne revient pas à Perlesvaus (mis à part celle qu'accomplit Lancelot p. 351), c'est la seule conversion dans la première partie du roman et elle précède les révélations hautement spirituelles du Château de l'Enquête; par ailleurs Gurgaran réapparaît plus loin, alors que les autres convertis disparaîtront définitivement de l'histoire (devenu ermite, il apporte à la cour d'Arthur une cloche sacrée). Gurgaran/ Archier finalement n'opère pas une conversion radicale: il y a plus transfert de croyance (translatio) que retournement radical.

Le cas du Chastel Tornoiant dans la branche IX est proche: d'après une prophétie, ce château, fondé par Virgile, cessera de tourner sur lui-même quand viendra un chevalier élu qui n'est autre que Perlesvaus (p. 250). Ces adeptes de la Viez Loi attendent le héros comme un Messie et se convertissent avec un empressement remarquable: le passage de l'Ancienne Loi à la Nouvelle a été différé, mais finalement il s'accomplit sans heurt, simple translatio espérée de longue date. Juste après, la reine au Cercle d'Or et son peuple, oppressés par le diabolique Chevalier au Dragon, sont libérés par Perlesvaus (p. 251-3): la victoire du héros sur le monstre atteste du pouvoir du Dieu chrétien et convainc sans peine la dame de se convertir. Comme Gurgaran, elle est pleine de bonne volonté, et elle fait à son sauveur un don qui tient de la relique en lui offrant le Cercle d'Or qui est en fait la corone d'espine (1. 4524). Ces conversion présentent des situations idéales: la conversion est une simple translatio fidei à partir d'un paganisme prémonitoire prenant volontiers le nom de Viez Loi, vers un christianisme espéré.

Les autres cas sont en revanche de véritables conversions dans un esprit de croisade impliquant des combats. Les conversions qui s'enchaînent alors sont de plus en plus difficiles et sélectives. A la Tour de Cuivre (p. 255) vit une gent mescreanz (1. 5939) endurcie dans son erreur: il croioient si fermement en cele male creance, que qui les vousist ocire en ce qu'il aoroient, si le sofrisent il, e quidasent estre sauf (1. 5944-ss). Leur culte est diabolique (le deiable en qui il croioient 1 . 5948) et renvoie à deux modèles, renforçant l'incertitude entre païens et juifs. 
L'adoration du Veau d'Or dénoncée dans l'Ancien Testament (Exode 32, 1-5) sert ici de modèle: on reconnaît l'abondance associée à ce culte: ( le deiable (...) lor donoit si grant habundance la dedenz que rien ne lor failoit 1. 5928-9), ainsi que les prophéties rendues par le monstre. La transposition romanesque transforme le veau, dont la jeunesse ne saurait servir les desseins de l'auteur, en taureau, plus inquiétant; à la place de l'or, connoté positivement, le cuivre traditionnellement lié à la rousseur maléfique renforce la dimension diabolique. Cependant avec son pont et ses automates armés de maillets de fer $(1.5927)^{63}$, ce lieu renvoie à l'espace des romans arthuriens ${ }^{64}$ et, comme le suggère Mary Williams, le culte du taureau, tel qu'il est décrit dans Perlesvaus, pourrait évoquer des pratiques datant de l'âge de Bronze $^{65}$, dont le souvenir aurait pu se perpétuer dans des récits de type folklorique. L'hésitation est donc entretenue entre judaïsme et paganisme préchrétien. Une voix miraculeuse ordonne à Perlesvaus de faire passer les habitants du château au milieu d'automates armés de marteaux: la porra il bien prover li quel vouront Deu croire e li quel non (1. 5953-4). Sur mille cinq cents, seuls treize survivront et se convertiront fermement et immédiatement (1. 5958). Désormais ce seront de vigilants défenseurs de la foi chrétienne: Josephez nos raconte que cil qui ces.XIII. bautisa ot non Denise, e li chausteax ot non li Chasteaux del Asai. Il furent la dedenz tant que la Novele Loi fu aseüree, e menerent mout bone vie; ne onques nus ne pot entrer avec aus que il ne fust ocis e detrenciés, se il ne creïst fermement en Deu. Quant cil de totes les isles orent ferme creance, li .XIII. qui ou chastel estoient s'en issirent fors, si firent hermitajes par les forez, por la fause loi que il avoient maintenue et por conquerre l'amor au Sauveor dou monde (1. 5966-ss). Cette conversion débouche sur un vif prosélytisme et une pénitence durable. Les figures ne sont pas individualisées, il n'est pas question de cheminement personnel qui conduirait à la conversion.

\footnotetext{
${ }^{63}$ Le Chastel Tornoiant est défendu par des archers de cuivre qui se tiennent aux créneaux; des lions et des ours enchaînés y breoient (p. 247): c'est un double de la Tour de Cuivre, protégée par ses homes faiz par l'art de nigromance armés de maus de fer et d'où s'échappent des hurlements comparables (La Tor de Cuevre... braoit 1. 5924).

${ }^{64}$ Sur les automates gardiens, voir notre livre Fées, bestes et luiton, Paris, PUPS, 2001, chap. VIII.

65 "The Episode of the Copper Tower in the Perlesvaus", dans Mélanges offerts à Rita Lejeune, Duculot, Gembloux, 1969, vol. II, p. 1159-1162.
} 
Dans la branche $\mathrm{X}$ de même les peuples conquis par Madaglan et convertis par celui-ci a la fausse creance (1. 8527) sont rapidement évoqués dans un épisode qui, selon une construction fréquente dans Perlesvaus, double celui de la Tor de Cuivre. Ces mescreant qui creoient es fausses ydres e es fauses ymages (1. 8536-7) sont des idolâtres. Conquis par Lancelot, ils sont confrontés à l'alternative épique sans que l'auteur s'attarde: li plusor se laisirent ocire por ce q'il ne voloient guerpir lor mauvese loi, e cil qui se voloient atorner a Dieu furent gari (p. 351). Lancelot brise les idoles et les remplace par des crucifix et des ymages a la senblance Nostre Saignor e a sa douce mere (p. 351).

Dans la branche XI, un nouveau type de conversion apparaît, avec le Chastel Enragié dont la dame devient chrétienne sous le nom de Celestre(p. 373) et avec Jandree et son peuple (p. 377). Dans un premier temps, les mescreant (1. 9115) du Chastel Enragié, des idolâtres d'un ancien temps, adorateurs de faux dieux qui ne sont que des diables (p. 369), sont victimes d'un miracle qui protège Perlesvaus (à la vue de ce bon chrétien, ils deviennent fous et s'entre-tuent) et tous ceux qui refusent la conversion sont passés par les armes. Plus nouveau dans Perlesvaus mais emprunté à la matière épique: la dame du lieu se convertit par amour (1. 9126-ss). Perlesvaus, dont la perfection est chaste, repousse les avances de la reine, mais finit par obtenir sa conversion en échange d'une promesse d'amour mal définie: "je vos amerai si come cil qui en Deu croient fermement doivent amer dames e damoiselles" (1. 9149-50). Perlesvaus la leva des sainz fons ; elle prend le nom de Celestre (p. 373). Un ermite reste près d'elle pour lui apprendre la fine creance (1. 9157). Cette expression fine creance n'est pas fréquente dans le texte: il suggère une idée de perfection (ce qui est conforté par la fin de la dame qui mènera désormais une vie molt sainte et fina puis en mout bones oevres 1. 9158-9). La fin'amors a conduit cette élue à la fine creance, l'amour courtois et l'amour de Dieu se rejoignant dans leurs fins. La conversion de la Reine Jandree, elle aussi soeur du païen Madaglan et mécréante, redouble celle de Celestre. Ayant entendu parler de Perlesvaus, de sa beauté et de sa valeur, la dame, rendue aveugle par sa mauvaise foi, souhaite recouvrer la vue pour pouvoir admirer ce héros (1. 9197-ss). Elle recouvre la vue par un miracle où Dieu et l'amour semblent se le disputer. En effet, lorsqu'elle prie ses Dieux de lui rendre la vue, ceux-ci l'informent qu'ils n'en ont pas le pouvoir. Elle se tourne alors vers le dieu de la Nouvelle Loi, qui lui accorde une vision où figurent la Trinité et la Crucifixion. 
Cette vision l'émeut et provoque sa pitié et ses larmes, et elle recouvre aussitôt la vue: elle impose la conversion, sous peine de mort, à tous ses gens. Elle se fait baptiser sous le nom symbolique de Salubre, fu molt bone dame, e bien creï en Dieu, e mena puis sainte vie qu'ele morut en un hermitage (p. 377). Cette conversion est aussi achevée que celle de Gurgaran: elle est l'occasion d'un catéchisme imagé et reprend la conception traditionnelle de la conversion émue ${ }^{66}$ et de la sainte fin. Comme dans le cas de sa soeur, en arrière-plan de la conversion, se devine un amour courtois, suscité par la réputation exceptionnelle du héros.

Les conversions confèrent au texte une progression: les trois premiers cas correspondent à des conversions de masse qui sont la réalisation d'une prophétie ou d'une attente (branche VI Gurgaran, branche IX le Chastel Tornoiant, le château de la Dame au Cercle d'Or); les autres (branche IX La Tour de Cuivre, branche X les peuples placés sous le joug de Madaglan, branche XI le Chastel Enragié et Jandree) sont l'occasion de conversions plus difficiles, au milieu d'une population hostile où les élus sont rares, les doublons étant nombreux (Gurgaran et le Chastel Tornoiant, la Tour de Cuivre et les païens de Madaglan, Celestre et Salubre). Loin de n'être qu'un patchwork de motifs hétéroclites, Perlesvaus serait donc structuré (entre autres) par le motif de la conversion, sa cohérence étant par ailleurs renforcée par deux images qui reviennent avec insistance: la conversion est rêvée à partir d'images circulaires et de figures de l'inversion.

Le vocabulaire de la conversion est fortement marqué par la circularité associée à deux racines: vertere et tornare. Le terme converti est utilisé par Perlesvaus dans le récit qu'il fait à son oncle de ses exploits au Chastel Tornoiant (1. 6043), atorné sert pour la conversion du Chastel Tornoiant, de Gurgaran et du Chastel Enragié ${ }^{67}$. Par ailleurs la conversion est associée à des espaces, des objets, des symboles circulaires et les déplacements des héros sont plus marqués par

\footnotetext{
${ }^{66}$ Sur ce don des larmes, voir J. C. Payen, op. cit., p. 31-ss et p. 37-ss. Arthur verse des larmes de contrition à la chapelle de Saint Augustin (p. 36).

${ }^{67}$ Perlesvaus (...) vit le pople du chastel atorné a la sainte loi (1. 5804); "je me fiz bautisier devant vos e tous ceaus de mon roiaume atorner a la Novele Loi" (1. 7237); Lanceloz (...) atorna a la loi Nostre Saingnor les mescreanz (1. 8541); atorner a sa loi (1. 9161).
} 
la circularité que par la linéarité: Perlesvaus ne sot quel part il vertiroit (1. 5920) ${ }^{68}$. La Demoiselle Chauve, dès le début du roman, introduit Fortune et sa roue, et les associe à la Crucifixion et donc à la conversion de l'Ancienne à la Nouvelle Loi, qui fonde l'Histoire dans Perlesvaus (p. 110). D'autres images, au fil du texte, reprennent l'association entre le cercle et la conversion. Le Chastel Tornoiant (p. 247-ss), fondé par Virgile, tourne jusqu'à sa conquête par le héros $^{69}$; l'enjeu associé à la conversion d'Elyza est le Cercle d'Or (p. 251). L'épisode de la Tor de Cuivre repose sur une hésitation entre le Tor et la Tor (la tor 1. 5939, 1. 5941, 1. 5860, le tor 1. 5922 , 1. 5959), et bien que les tours ne soient pas nécessairement rondes, il semble que l'homonymie entre tor ("turris") et tor ("torner") favorise dans l'épisode l'image d'un bâtiment rond $^{70}$, d'autant que la racine virer ${ }^{71}$ est très présente dans l'épisode (environ 1. 5925, 1. 5931, 1. 5941 avironné 1. 5940, avironne 1. 5954). Par ailleurs, c'est la Table Ronde que réclame Madaglan à Arthur en même temps que sa conversion (p. 327) et les châteaux des impies sont souvent fermés par des ponts tournants (c'est le cas du Chastel Enragié $1.9067^{72}$ et de celui du Noir Ermite 1. 9967).

A côté de cette image très présente, d'autres, plus discrètes, peuvent être mises en évidence, comme celle de l'inversion. Le Chevalier Couard qui chevauche à l'envers est une allégorie de l'Ancienne Loi. La loi estoit bestornee devant le crucefiement Nostre Saingnor (1. 2217) et la Crucifixion l'a remise a droit (1. 2218): de même le Chevalier Couard devient Hardi (p. 111, p. 359). Cette image de retournement, moins présente que les figures circulaires, n'est pas sans lien avec ces dernières: tout retournement s'inscrit dans un cercle. Or ces images de la conversion sont révélatrices des limites de celle-ci. Le cercle revient à son point de départ, le

\footnotetext{
${ }^{68}$ Sur la circularité de l'itinéraire de Gauvain, voir T.E . Kelly, op. cit., p. 64.

${ }^{69}$ Sur le château qui tourne, voir notre Fées, bestes et luitons, op. cit., chap. III.

${ }^{70}$ On peut hésiter avec Ch. Méla entre cor et tor, les graphies /t/ et /c/ étant facilement confondues (La reine et le Graal, Paris, Seuil, 1984, p. 443). Cependant il me semble qu'il ne faut pas rejeter la forme tor afin de conserver l'ambiguité entre "la tor" et "le tor".

${ }^{71}$ Virer est d'abord attesté avec l'idée de "lancer en faisant tourner".

${ }^{72}$ La présentation qui est faite du Chastel Enragié insiste sur la circularité: .I. grant chastel (...) a grant pont torneiz, e avoit grant tour (1. 9068-9).
} 
retournement peut annuler le déplacement: la conversion est réversible et donc incertaine, comme le montre l'étude de ses limites.

La foi dans Perlesvaus est sans cesse à renouveler et restaurer (1. 7601). Les renégats sont légion (1. 6115). Les habitants de la terre du Roi Pécheur ont deguerpie ${ }^{73}$ la Novele Loi (1. 6056) sous la contrainte du Roi du Chastel Mortel. Les païens ne cessent de provoquer les Chrétiens à l'apostasie, qu'il s'agisse de Madaglan (1. 7849) ou de Jandrée (1. 9142) et Madaglan a une conception de la conversion digne d'un croisé (toz çaus qui ne voloient croire en lor diex faisoient morir e trenchoient les testes 1.8147$)^{74}$.

Outre que les conversions sont incertaines (soumises au mouvement de la Roue de Fortune symbolisée par la Demoiselle Chauve), il semble que le texte les escamote souvent. L'épisode de la nef est très significatif. Après la conversion de Dame Salubre, Perlesvaus quitte, grâce à l'entrelacement, le devant de la scène. Meliot tue alors le chevalier de la Galie, dont nous ne saurons quasiment rien, puis sauve Gauvain: tous deux voient alors de loin une nef (p. 382). Gauvain annonce, sans que l'on sache d'où lui vient cette information, que Perlesvaus est en danger sur cette embarcation et que s'il périt, aucun autre chevalier n'avra si grant pooir d'essaucier la loi Nostre Saignor (p. 382). Rien n'a introduit dans le texte cette nef, beaucoup plus animée que les nefs mystiques qui voguent dans la Queste del Saint Graal, appelée tout au plus par le nom du Chevalier de la Galie et par le fait que les sarrasins étaient, à juste titre, réputés bons marins. Le texte se désintéresse alors rapidement de ce navire, pour parler de Claudas, Arthur et Lancelot. Il revient néanmoins plus loin à Perlesvaus resté sur son bateau et victorieux de ses adversaires (p. 387): on apprend alors que le héros a tué tous les passagers de la nef, fors cel qui le gouverne, quar il li a en covenant que il crera en Deu et guerpira sa mauvese loi (1. 9539). Le texte, grâce à l'entrelacement, a éludé une scène de conversion qui a tourné, selon

\footnotetext{
73 L'expression guerpir la Novele Loi est fréquente (1. 7849, 1. 7934, 1. 8495). Ce verbe guerpir est péjoratif et marque que la loi rejetée est mauvaise (dans la bouche de Perceval il renvoie à la mauvaise foi païenne, 1. 9141, dans la bouche de païens, il renvoie à la Nouvelle Loi 1. 7849, 1. 7934).

${ }^{74}$ Perlesvaus fait la même chose avec ceux qui, après la défaite du roi de Chastel Mortel, refusent de se convertir (l. $6259)$.
} 
l'alternative épique, au massacre général, mis à part le pilote. Cette exception est curieuse, même si elle va dans le sens de toutes les autres conversions sélectives de Perlesvaus (songeons aux treize païens de la Tour de Cuivre). En fait, dans le cas de la nef, l'exception permet peut-être surtout à Perlesvaus d'avoir un timonier fiable: l'auteur de Perlesvaus, contrairement à celui de $\mathrm{La}$ Queste del Saint Graal use avec discrétion des miracles et ne nous invente pas de gracieuses nefs automobiles. Il aurait certes pu recourir à ce type de navigation mystique, puisqu'il nous explique que c'est Dieu qui guide le navire (1.9542), mais il préfère convertir un timonier: esprit positif, il prêche un idéal de croisade et d'action, et ne nourrit pas ses lecteurs d'images rassurantes. Par ailleurs, cette unique conversion est tout à fait représentative du fait que dans Perlesvaus, le sort normalement réservé au païen est la mort, à quelques rares exceptions près. C'est donc un idéal militant et guerrier qui se dégage, plus proche des chansons de geste que d'un roman comme $L a$ Queste del Saint Graal.

Plus que les peuples, ce sont les lieux qui passent à Dieu: si la conversion reste marginale, la consécration de l'espace conquis est en revanche toujours un succès. Des églises et des ermitages sont fondés, sans que le texte pourtant s'attarde longuement sur les détails. La conversion du Château du Noir Ermite est escamotée, bien que ce soit l'ultime épreuve, celle où le héros est confrontée au Diable: une fois le seigneur jeté dans l'abîme infernal, Perlesvaus se parti du chastel joianz, car il l'ot atret a la creance Nostre Seigneur, et fist on son servise dedenz autressi saintement com es autres lex (1. 10001-3). L'espace a été rendu au culte, sans qu'il semble y avoir eu beaucoup de conversions, mais l'information n'est donnée que rétrospectivement. Souvent par ailleurs, les expressions sont ambiguës, et l'on ne sait pas s'il y a eu conversion ou massacre: évoquant Lancelot qui a converti la terre du roi d'Oriande, au grand dam de ses soeurs, le texte utilise une formule incertaine: il avoit la fause gent ostee (1. 9065). Les quelques conversions avérées et les nouveaux lieux consacrés n'empêchent pas que l'extermination prédomine dans Perlesvaus: le roi Ermite, glosant la Beste Glatissant à Perlesvaus, utilise un futur qui ne laisse guère d'espoir: li Juïfs qui sauvaje sunt e ierent d'ore en avant (1. 6005-6). W. A. Nitze en conclut qu'on peut opposer un Perlesvaus clunisien à une Queste del Saint Graal 
cistercienne, les clunisiens prêchant plus l'extermination que la conversion des infidèles ${ }^{75}$. JeanCharles Payen y voit plutôt une dimension épique, absente de la Queste ${ }^{76}$. Le fait que les "conversions" sont plus de l'ordre de l'image obsessionnelle que de la réflexion spirituelle m'orienterait de même vers un Perlesvaus plus épique que clunisien si un certain nombre d'options du texte n'étaient pas incompatibles, me semble-t-il, avec le monde des chansons de geste. En effet, Perlesvaus ne semble pas sans remords face à la violence qui bafoue les valeurs chevaleresques fondamentales. Lorsque les chevaliers qui gardent le Chastel Mortel implorent merci (p. 266), Perlesvaus les massacre, mais il ne prend pas seul la décision de les tuer: il regarde d'abord le lion blanc qui garde l'entrée et dont le roi Ermite lui a expliqué qu'il permet de reconnaître les pensées inspirées par Dieu, qui donc cautionne l'acte du héros. L'auteur ne semble guère croire en la valeur du prêche et de l'exemple, et c'est avec une relative mauvaise conscience qu'il évoque les massacres: l'espoir réside finalement dans l'autodestruction des forces du Mal, et l'adversaire suprême, le Noir Ermite, périt, jeté dans l'abîme par les siens (1. 9988), tandis que le dragon du Chevalier au Dragon se rebelle contre son maitre et le calcine. On est loin de la suffisance du héros épique face au Mal. Par ailleurs, l'auteur de Perlesvaus, qui ne croit guère en la conversion, semble viser moins la conversion des mécréants que le repentir des Chrétiens, qu'il s'agisse du pèlerinage d'Arthur (p. 269, p. 274) ou de la pénitence de la demoiselle du Château des Barbes (p. 276-7), dans un mouvement de retour sur soi qui me semble n'avoir rien d'épique. Comme l'a montré Jean-Charles Payen, la pénitence est un thème essentiel dans ce roman: il est introduit avec l'ermite qui a vécu plus de soixante ans dans le péché et n'a entrepris sa pénitence que cinq ans avant de mourir et avec la Demoiselle Chauve: "le monde arthurien (et le roi en particulier) s'est tourné vers les plaisirs au lieu d'évangéliser une Bretagne encore à demi païenne" ${ }^{77}$. Contrairement au Merlin néanmoins, Perlesvaus, en dépit de cet intérêt pour la pénitence et la conversion, ne montrera pas un monde arthurien racheté: les conceptions d'Arthur et Merlin restent douteuses (p. 281-2) et même le repentir, moins ambitieux que la conversion,

\footnotetext{
${ }^{75}$ Voir l'édition, t. II, p. 86-ss.

${ }^{76}$ Op. cit., p. 423.

${ }^{77}$ Ibid., p. 421.
} 
reste en suspens ${ }^{78}$. Perlesvaus a renoncé à convertir les païens ${ }^{79}$, et, hanté par des déviances, des translations incertaines, celle des Juifs qui n'ont pas suivi la conversion historique, celle du monde arthurien, dont l'origine n'est pas sans tâche et qui semble miné par la décadence (1. 70-ss), il rappelle les grandes vérités nécessaires aux Croisés et à tout bon chrétien (la Trinité, l'Incarnation, l'Eucharistie, la Rédemption). Si dans les chansons de geste, le païen reste estrange, dans les romans arthuriens, l'enjeu de la conversion a tendance à se rapprocher (ne serait-ce qu'à cause de la géographie imposée par le genre): d'une part le païen appelé à se convertir n'est plus l'étranger absolu, il peut être un ancêtre, un chrétien renégat ; d'autre part les Chrétiens doivent sans cesse être confortés dans leur foi, le paganisme, comme dans Perceforest, resurgissant sans cesse et maintenant toujours des poches de résistances, souvent insulaires. Les aléas des croisades extérieures et la lutte contre les hérétiques ont certainement joué un rôle dans cette vision, mais il me semble aussi que l'on peut voir dans cet échec des conversions dans Perlesvaus la marque d'un faux départ de la prose romanesque.

Bâti, depuis les adaptations du XIIe siècle, sur la puissance conception de la translatio studii, le roman s'est mis au service d'une historiographie fondée sur la translatio imperii qui, le Graal aidant, s'est doublée d'une translatio fidei. Perceforest au XIVe siècle est un exemple particulièrement évocateur de cette imbrication des trois translationes. Perlesvaus, plus d'un siècle plus tôt, présente lui aussi un héros chargé d'assurer le passage (à travers vaux et forêts), mais il reste sceptique devant toute translatio. Si Merlin travaille avec succès à la Rédemption du monde arthurien et simultanément au cautionnement de ce genre à la légitimité douteuse qu'est le roman en prose, Perlesvaus, vraisemblablement à peu près à la même époque, sape les fondements du monde arthurien en limitant les chances de succès de la pénitence royale et en ne rachetant pas explicitement Merlin, et il limite par là même les effets des translationes, religieuses ou littéraires. N'assurant guère de fondement solide au roman en prose arthurien,

\footnotetext{
${ }^{78}$ Voir notre article, "La parole dans le Merlin de Robert de Boron", dans Merlin, ouvrage dirigé par D. Quéruel et C. Ferlampin-Acher, Paris, Ellipses, 2000, p. 89-104.

${ }^{79}$ Voir T. E. Kelly, op. cit., p. 96.
} 
contrairement au Merlin, il ne semble pas avoir eu de descendance, ce qui est étonnant pour un texte ancien. Comme le conclut F. Dubost à partir d'une autre voix, nocturne, Perlesvaus est un $\operatorname{anti-roman}^{80}$, sans descendance ni ascendance. Si la tradition arthurienne s'enracine dans des démarches complexes qui inventent des sources authentiques et spéculaires ${ }^{81}$, Perlesvaus au contraire ne se présente pas comme une récriture, une translatio: il tient de la révélation, immédiate, transmise par la voix d'un ange $(1.4)^{82}$ : il échappe d'emblée à la linéarité rassurante de l'emprunt et de la culture, de l'héritage, et opte pour l'immédiateté de la révélation, en dépit des renvois traditionnels à l'escriture (par exemple 1. 58). Invalidant la translatio, il se condamne à rester sans postérité. Placé sous le signe de la perte et non du passage (Perlesvaus est plus celui qui perd les vaux, que celui qui les traverse), ce roman, glissant de per/par à per/perd, renonce à la possession, à l'héritage, à la transmission, à la translatio et à la postérité littéraire: il se fait fulgurance ${ }^{83}$ brutale et brehaigne.

\footnotetext{
80 "Les nuits magnétiques du Perlesvaus", dans Miscellanea Mediaevalia, op. cit., t. I, p. 429-ss, en particulier p. 445 .

${ }^{81}$ Pensons en particulier à l'Estoire del Saint Graal et à son prologue d'une complexité remarquable: voir A. Leupin, Le Graal et la littérature, Lausanne, L'âge d'homme, 1982, p. 24-ss.

${ }^{82}$ Sur Perlesvaus et la voix de l'ange, voir Ch. Méla, op. cit., p. 176.

${ }^{83}$ Voir 1. 301-ss.
} 